\title{
Lyapunov matrices approach to the parametric optimization of a system with two delays
}

\author{
JOZEF DUDA
}

In the paper a Lyapunov matrices approach to the parametric optimization problem of time-delay systems with two commensurate delays and a P-controller is presented. The value of integral quadratic performance index of quality is equal to the value of the Lyapunov functional for the initial function of time-delay system. The Lyapunov functional is determined by means of the Lyapunov matrix.

Key words: time-delay system, Lyapunov matrix, Lyapunov functional.

\section{Introduction}

The Lyapunov functionals are used to test the stability of systems, in calculation of the robustness bounds for uncertain time delay systems, in computation of the exponential estimates for the solutions of time delay systems. The method of determination of a Lyapunov functional for a time delay system with one delay, for the first time, was presented by Repin [13]. Duda used the Repin's method to determination of a Lyapunov functional for a system with two delays [2], for a neutral system [3], for a system with a time-varying delay $[4,5]$, for a neutral system with both lumped and distributed delay [7].

The Lyapunov quadratic functionals are also used to calculation of a value of a quadratic performance index of quality in the process of the parametric optimization for time delay systems. One constructs a functional for a system with a time delay with a given time derivative whose is equal to the negatively defined quadratic form of a system state. The value of that functional at the initial state of a time delay system is equal to the value of a quadratic performance index of quality.

In last years a method of determination of the Lyapunov functional by means of Lyapunov matrices is very popular, see for example [10-12,14]. Duda used a method of determination of the Lyapunov functional by means of Lyapunov matrix in the parametric optimization problem to a time-delay system with one delay [6] and to a neutral

The Author is with AGH University of Science and Technology, Department of Automatics and Biomedical Engineering, Krakow, Poland. E-mail: jduda@ agh.edu.pl.

Received 24.02.2016. 
system with one delay [8]. In this paper is presented a Lyapunov matrices approach to the parametric optimization problem of a time-delay system with two delays. This paper extends results of [6] to the time delay system with two delays.

\section{Preliminaries}

Let us consider a time-delay system

$$
\left\{\begin{array}{l}
\frac{d x(t)}{d t}=\sum_{j=0}^{m} A_{j} x\left(t-h_{j}\right) \\
x\left(t_{0}+\theta\right)=\varphi(\theta)
\end{array}\right.
$$

for $t \geqslant t_{0}, \theta \in[-h, 0]$, where $x(t) \in \mathbb{R}^{n}, A_{j} \in \mathbb{R}^{n \times n}, 0=h_{0}<h_{1}<\ldots<h_{m}=h$, function $\varphi \in P C\left([-h, 0], \mathbb{R}^{n}\right)$ - the space of piece-wise continuous vector valued functions defined on the segment $[-h, 0]$ with the uniform norm $\|\varphi\|_{P C}=\sup _{\theta \in[-h, 0]}\|\varphi(\theta)\|$.

The theorems of existence, continuous dependence and uniqueness of solutions of equation (1) are given in [9].

Let $x\left(t, t_{0}, \varphi\right)$ be the solution of system (1) with the initial function $\varphi$.

$K(t)$ is the fundamental matrix of system (1) if it satisfies the matrix equation

$$
\frac{d}{d t} K(t)=\sum_{j=0}^{m} A_{j} K\left(t-h_{j}\right)
$$

for $t \geqslant 0$ and the following initial condition $K(0)=I_{n \times n}$ and $K(t)=0_{n \times n}$ for $t<0$ where $I_{n \times n}$ is the identity $n \times n$ matrix and $0_{n \times n}$ is the zero $n \times n$ matrix.

Theorem 1 (Bellman \& Cooke [1]). Let $K(t)$ be the fundamental matrix of system (1), then for $t \geqslant t_{0}$

$$
x\left(t, t_{0}, \varphi\right)=K\left(t-t_{0}\right) \varphi(0)+\sum_{j=1}^{m} \int_{-h_{j}}^{0} K\left(t-t_{0}-h_{j}-\theta\right) A_{j} \varphi(\theta) d \theta
$$

The function $x_{t}\left(t_{0}, \varphi\right):[-h, 0] \rightarrow \mathbb{R}^{n}$ is called a shifted restriction of $x\left(\cdot, t_{0}, \varphi\right)$ to an interval $[t-h, t]$ and is defined by a formula

$$
x_{t}\left(t_{0}, \varphi\right)(\theta):=x\left(t+\theta, t_{0}, \varphi\right)
$$

for $t \geqslant t_{0}$ and $\theta \in[-h, 0]$.

The initial condition holds

$$
x_{t_{0}}\left(t_{0}, \varphi\right)=\varphi
$$

for $\theta \in[-h, 0]$. 


\section{A Lyapunov-Krasovskii functional}

Given a symmetric positive definite matrix $W \in \mathbb{R}^{n \times n}$. We are looking for a functional $v: P C\left([-h, 0], \mathbb{R}^{n}\right) \rightarrow \mathbb{R}$ such that along the solutions of system (1) the following equality holds

$$
\frac{d}{d t} v\left(x_{t}\left(t_{0}, \varphi\right)\right)=-x^{T}\left(t, t_{0}, \varphi\right) W x\left(t, t_{0}, \varphi\right)
$$

for $t \geqslant t_{0}$, where $x\left(t, t_{0}, \varphi\right)$ is a solution of system (1), with the initial function $\varphi \in$ $P C\left([-h, 0], \mathbb{R}^{n}\right)$, given by (2).

We assume that system (1) is asymptotically stable and integrate both side of Eq. (5) from $t_{0}$ to infinity. We obtain

$$
v\left(x_{t_{0}}\left(t_{0}, \varphi\right)\right)=v(\varphi)=\int_{t_{0}}^{\infty} x^{T}\left(t, t_{0}, \varphi\right) W x\left(t, t_{0}, \varphi\right) d t
$$

Taking into account (2) we calculate the integral of the right-hand side of Eq. (6)

$$
\begin{gathered}
\int_{t_{0}}^{\infty} x^{T}\left(t, t_{0}, \varphi\right) W x\left(t, t_{0}, \varphi\right) d t=\varphi^{T}(0) \int_{0}^{\infty} K^{T}(t) W K(t) d t \varphi(0)+ \\
+\sum_{j=1}^{m} \int_{-h_{j}}^{0} 2 \varphi^{T}(0) \int_{0}^{\infty} K^{T}(t) W K\left(t-h_{j}-\theta\right) d t A_{j} \varphi(\theta) d \theta+ \\
+\sum_{j=1}^{m} \sum_{k=1}^{m} \int_{-h_{j}}^{0} \varphi^{T}(\theta) A_{j}^{T} \int_{-h_{k}}^{0} \int_{0}^{\infty} K^{T}\left(t-h_{j}-\theta\right) W K\left(t-h_{k}-\eta\right) d t A_{k} \varphi(\eta) d \eta d \theta
\end{gathered}
$$

The following relations hold

$$
\begin{gathered}
\int_{0}^{\infty} K^{T}\left(t-h_{j}-\theta\right) W K\left(t-h_{k}-\eta\right) d t=\int_{-h_{j}-\theta}^{\infty} K^{T}(\varsigma) W K\left(\varsigma+h_{j}-h_{k}+\theta-\eta\right) d \varsigma= \\
=\int_{-h_{j}-\theta}^{0} K^{T}(\varsigma) W K\left(\varsigma+h_{j}-h_{k}+\theta-\eta\right) d \varsigma+\int_{0}^{\infty} K^{T}(\varsigma) W K\left(\varsigma+h_{j}-h_{k}+\theta-\eta\right) d \varsigma= \\
=\int_{0}^{\infty} K^{T}(\varsigma) W K\left(\varsigma+h_{j}-h_{k}+\theta-\eta\right) d \varsigma
\end{gathered}
$$


The term

$$
\int_{-h_{j}-\theta}^{0} K^{T}(\varsigma) W K\left(\varsigma+h_{j}-h_{k}+\theta-\eta\right) d \varsigma=0
$$

because $K(\varsigma)=0$ for $\varsigma<0$. Formula (7) takes a form

$$
\begin{gathered}
\int_{t_{0}}^{\infty} x^{T}\left(t, t_{0}, \varphi\right) W x\left(t, t_{0}, \varphi\right) d t=\varphi^{T}(0) \int_{0}^{\infty} K^{T}(t) W K(t) d t \varphi(0)+ \\
+\sum_{j=1}^{m} \int_{-h_{j}}^{0} 2 \varphi^{T}(0) \int_{0}^{\infty} K^{T}(t) W K\left(t-h_{j}-\theta\right) d t A_{j} \varphi(\theta) d \theta+ \\
+\sum_{j=1}^{m} \sum_{k=1}^{m} \int_{-h_{j}}^{0} \varphi^{T}(\theta) A_{j}^{T} \int_{-h_{k}}^{0} \int_{0}^{\infty} K^{T}(\varsigma) W K\left(\varsigma+h_{j}-h_{k}+\theta-\eta\right) d \varsigma A_{k} \varphi(\eta) d \eta d \theta
\end{gathered}
$$

We introduce a Lyapunov matrix

$$
U(\xi)=\int_{0}^{\infty} K^{T}(t) W K(t+\xi) d t
$$

for $\xi \geqslant 0$. Using the Lyapunov matrix (9) and taking into account Eq. (6) we obtain a formula for a functional $v(\varphi)$

$$
\begin{aligned}
& v(\varphi)= \int_{t_{0}}^{\infty} x^{T}\left(t, t_{0}, \varphi\right) W x\left(t, t_{0}, \varphi\right) d t=\varphi^{T}(0) U(0) \varphi(0)+ \\
&+2 \varphi^{T}(0) \sum_{j=1}^{m} \int_{-h_{j}}^{0} U\left(-\theta-h_{j}\right) A_{j} \varphi(\theta) d \theta+ \\
&+\sum_{j=1}^{m} \sum_{k=1}^{m} \int_{-h_{j}}^{0} \int_{-h_{k}}^{0} \varphi^{T}(\theta) A_{j}^{T} U\left(h_{j}-h_{k}+\theta-\eta\right) A_{k} \varphi(\eta) d \eta d \theta
\end{aligned}
$$

Theorem 2 The Lyapunov matrix (9) satisfies the following properties [14]:

Dynamic property

$$
\frac{d}{d \xi} U(\xi)=\sum_{j=0}^{m} U\left(\xi-h_{j}\right) A_{j}
$$

for $\xi \geqslant 0$. 
Symmetry property

$$
U(-\xi)=U^{T}(\xi)
$$

for $\xi \geqslant 0$.

\section{Algebraic property}

$$
\sum_{j=0}^{m}\left[U\left(-h_{j}\right) A_{j}+A_{j}^{T} U\left(h_{j}\right)\right]=-W
$$

Formulas (11), (12), (13) enable us to calculate the Lyapunov matrix $U(\xi)$ for $\xi \geqslant 0$.

\section{A Lyapunov matrix for a system with two commensurate delays}

Let us consider the following system

$$
\left\{\begin{array}{l}
\frac{d x(t)}{d t}=A_{0} x(t)+A_{1} x(t-h)+A_{2} x(t-2 h) \\
x(\theta)=\varphi(\theta)
\end{array}\right.
$$

for $t \geqslant 0$ and $\theta \in[-2 h, 0]$. Where $A_{0}, A_{1}, A_{2} \in \mathbb{R}^{n \times n}$ and $\varphi \in P C\left([-h, 0], \mathbb{R}^{n}\right), 0<h \in \mathbb{R}$.

A set of equations (11), (12), (13) for system (14) takes a form

$$
\begin{gathered}
\frac{d}{d \xi} U(\xi)=U(\xi) A_{0}+U(\xi-h) A_{1}+U(\xi-2 h) A_{2} \\
U(-\xi)=U^{T}(\xi) \\
U(0) A_{0}+U(-h) A_{1}+U(-2 h) A_{2}+A_{0}^{T} U(0)+A_{1}^{T} U(h)+A_{2}^{T} U(2 h)=-W
\end{gathered}
$$

for $\xi \in[0,2 h]$. The relation (16) implies

$$
U(-h)=U^{T}(h) \text { and } U(-2 h)=U^{T}(2 h)
$$

so we can write Eq. (17) in a form

$$
U(0) A_{0}+U^{T}(h) A_{1}+U^{T}(2 h) A_{2}+A_{0}^{T} U(0)+A_{1}^{T} U(h)+A_{2}^{T} U(2 h)=-W
$$

Formula (16) extends the function $U$ defined on the segment $[0,2 h]$ to the segment $[-2 h, 0]$. Indeed for $\xi \in[0,2 h], U(-\xi)=U^{T}(\xi)$. For $\tau=-\xi, U(\tau)=U^{T}(-\tau)$ and $\tau \in[-2 h, 0]$. 
We define the functions $U_{1}(\xi), U_{2}(\xi), Z_{1}(\xi), Z_{2}(\xi)$ for $\xi \in[0, h]$

$$
\begin{gathered}
U_{1}(\xi)=U(\xi) \\
U_{2}(\xi)=U(h+\xi) \\
Z_{1}(\xi)=U(\xi-h)=U^{T}(-\xi+h) \\
Z_{2}(\xi)=U(\xi-2 h)=U^{T}(-\xi+2 h)
\end{gathered}
$$

For $\xi \in[0, h]$ Eq. (15) can be written in a form

$$
\frac{d}{d \xi} U_{1}(\xi)=U_{1}(\xi) A_{0}+Z_{1}(\xi) A_{1}+Z_{2}(\xi) A_{2}
$$

For $\xi+h=\varsigma \in[h, 2 h]$

$U(\varsigma)=U(\xi+h)=U_{2}(\xi), U(\varsigma-h)=U(\xi)=U_{1}(\xi), U(\varsigma-2 h)=U(\xi-h)=Z_{1}(\xi)$

and Eq. (15) can be written in a form

$$
\frac{d}{d \xi} U_{2}(\xi)=U_{2}(\xi) A_{0}+U_{1}(\xi) A_{1}+Z_{1}(\xi) A_{2}
$$

We compute the derivative of $Z_{1}(\xi)$

$$
\begin{gathered}
\frac{d}{d \xi} Z_{1}(\xi)=\frac{d}{d \xi} U^{T}(-\xi+h)=\frac{d}{d \tau} U^{T}(\tau) \frac{d \tau}{d \xi}=-\frac{d}{d \tau} U^{T}(\tau)= \\
=-A_{0}^{T} U^{T}(\tau)-A_{1}^{T} U^{T}(\tau-h)-A_{2}^{T} U^{T}(\tau-2 h)= \\
=-A_{0}^{T} U^{T}(-\xi+h)-A_{1}^{T} U^{T}(-\xi)-A_{2}^{T} U^{T}(-\xi-h)= \\
=-A_{0}^{T} Z_{1}(\xi)-A_{1}^{T} U_{1}(\xi)-A_{2}^{T} U_{2}(\xi)
\end{gathered}
$$

where $\tau=-\xi+h$ and the derivative of $Z_{2}(\xi)$

$$
\begin{gathered}
\frac{d}{d \xi} Z_{2}(\xi)=\frac{d}{d \xi} U^{T}(-\xi+2 h)=\frac{d}{d \tau} U^{T}(\tau) \frac{d \tau}{d \xi}=-\frac{d}{d \tau} U^{T}(\tau)= \\
=-A_{0}^{T} U^{T}(\tau)-A_{1}^{T} U^{T}(\tau-h)-A_{2}^{T} U^{T}(\tau-2 h)= \\
=-A_{0}^{T} U^{T}(-\xi+2 h)-A_{1}^{T} U^{T}(-\xi+h)-A_{2}^{T} U^{T}(-\xi)= \\
=-A_{2}^{T} U_{1}(\xi)-A_{1}^{T} Z_{1}(\xi)-A_{0}^{T} Z_{2}(\xi)
\end{gathered}
$$

and where $\tau=-\xi+2 h$. 
We have received a set of ordinary differential equations

$$
\left\{\begin{array}{l}
\frac{d}{d \xi} U_{1}(\xi)=U_{1}(\xi) A_{0}+Z_{1}(\xi) A_{1}+Z_{2}(\xi) A_{2} \\
\frac{d}{d \xi} U_{2}(\xi)=U_{1}(\xi) A_{1}+U_{2}(\xi) A_{0}+Z_{1}(\xi) A_{2} \\
\frac{d}{d \xi} Z_{1}(\xi)=-A_{1}^{T} U_{1}(\xi)-A_{2}^{T} U_{2}(\xi)-A_{0}^{T} Z_{1}(\xi) \\
\frac{d}{d \xi} Z_{2}(\xi)=-A_{2}^{T} U_{1}(\xi)-A_{1}^{T} Z_{1}(\xi)-A_{0}^{T} Z_{2}(\xi)
\end{array}\right.
$$

for $\xi \in[0, h]$ with initial conditions

$$
U_{1}(0), U_{2}(0), Z_{1}(0), Z_{2}(0)
$$

The following relations hold

$$
U(0)=U_{1}(0), U(h)=U_{2}(0), U(2 h)=U_{2}(h)
$$

and therefore Eq. (18) takes a form

$$
U_{1}(0) A_{0}+U_{2}^{T}(0) A_{1}+U_{2}^{T}(h) A_{2}+A_{0}^{T} U_{1}(0)+A_{1}^{T} U_{2}(0)+A_{2}^{T} U_{2}(h)=-W
$$

Using the Kronecker product we can express Eq. (27) in a form

$$
\left[\begin{array}{c}
\frac{d}{d \xi} \operatorname{col}_{1}(\xi) \\
\frac{d}{d \xi} \operatorname{col}_{2}(\xi) \\
\frac{d}{d \xi} \operatorname{colZ}_{1}(\xi) \\
\frac{d}{d \xi} \operatorname{colZ}_{2}(\xi)
\end{array}\right]=\mathcal{H}\left[\begin{array}{l}
\operatorname{col} U_{1}(\xi) \\
\operatorname{col} U_{2}(\xi) \\
\operatorname{colZ}(\xi) \\
\operatorname{colZ}(\xi)
\end{array}\right]
$$

for $\xi \in[0, h]$ with initial conditions

$$
\operatorname{col}_{1}(0), \operatorname{col}_{2}(0), \operatorname{col}_{1}(0), \operatorname{col}_{2}(0)
$$

where

$$
\mathcal{H}=\left[\begin{array}{cccc}
A_{0}^{T} \otimes I & 0 & A_{1}^{T} \otimes I & A_{2}^{T} \otimes I \\
A_{1}^{T} \otimes I & A_{0}^{T} \otimes I & A_{2}^{T} \otimes I & 0 \\
-I \otimes A_{1}^{T} & -I \otimes A_{2}^{T} & -I \otimes A_{0}^{T} & 0 \\
-I \otimes A_{2}^{T} & 0 & -I \otimes A_{1}^{T} & -I \otimes A_{0}^{T}
\end{array}\right]
$$


Formula (28) can be expressed in a form

$$
\begin{gathered}
\left(A_{0}^{T} \otimes I+I \otimes A_{0}^{T}\right) \operatorname{col}_{1}(0)+\left(I \otimes A_{1}^{T}\right) \operatorname{col}_{2}(0)+ \\
+\left(A_{1}^{T} \otimes I\right) \operatorname{col}_{2}^{T}(0)+\left(I \otimes A_{2}^{T}\right) \operatorname{col}_{2}(h)+\left(A_{2}^{T} \otimes I\right) \operatorname{col}_{2}^{T}(h)=-\operatorname{col} W
\end{gathered}
$$

Solution of Eq. (29) is given in the form

$$
\left[\begin{array}{c}
\operatorname{col}_{1}(\xi) \\
\operatorname{col}_{2}(\xi) \\
\operatorname{colZ}_{1}(\xi) \\
\operatorname{colZ}_{2}(\xi)
\end{array}\right]=\Phi(\xi)\left[\begin{array}{c}
\operatorname{colU}_{1}(0) \\
\operatorname{colU}_{2}(0) \\
\operatorname{colZ}_{1}(0) \\
\operatorname{colZ}_{2}(0)
\end{array}\right]
$$

where a matrix

$$
\Phi(\xi)=\left[\begin{array}{llll}
\Phi_{11}(\xi) & \Phi_{12}(\xi) & \Phi_{13}(\xi) & \Phi_{14}(\xi) \\
\Phi_{21}(\xi) & \Phi_{22}(\xi) & \Phi_{23}(\xi) & \Phi_{24}(\xi) \\
\Phi_{31}(\xi) & \Phi_{32}(\xi) & \Phi_{33}(\xi) & \Phi_{34}(\xi) \\
\Phi_{41}(\xi) & \Phi_{42}(\xi) & \Phi_{43}(\xi) & \Phi_{44}(\xi)
\end{array}\right]
$$

is a fundamental matrix of system (29).

We determine the initial conditions

$$
\operatorname{col}_{1}(0), \operatorname{col}_{2}(0), \operatorname{col}_{1}(0), \operatorname{colZ}_{2}(0)
$$

From Eq. (31) we obtain

$$
\begin{gathered}
\operatorname{col}_{1}(h)=\operatorname{col}_{2}(0)=\Phi_{11}(h) \operatorname{col}_{1}(0)+ \\
+\Phi_{12}(h) \operatorname{col}_{2}(0)+\Phi_{13}(h) \operatorname{col}_{1}(0)+\Phi_{14}(h) \operatorname{col}_{2}(0) \\
\operatorname{colZ}_{1}(h)=\operatorname{colU}_{1}(0)=\Phi_{31}(h) \operatorname{col}_{1}(0)+ \\
+\Phi_{32}(h) \operatorname{col}_{2}(0)+\Phi_{33}(h) \operatorname{col}_{1}(0)+\Phi_{34}(h) \operatorname{col}_{2}(0) \\
\operatorname{colZ}_{2}(h)=\operatorname{colZ}_{1}(0)=\Phi_{41}(h) \operatorname{col}_{1}(0)+ \\
+\Phi_{42}(h) \operatorname{col}_{2}(0)+\Phi_{43}(h) \operatorname{col}_{1}(0)+\Phi_{44}(h) \operatorname{col}_{2}(0) \\
\operatorname{col}_{2}(h)=\Phi_{21}(h) \operatorname{col}_{1}(0)+\Phi_{22}(h) \operatorname{col}_{2}(0)+ \\
+\Phi_{23}(h) \operatorname{col}_{1}(0)+\Phi_{24}(h) \operatorname{colZ}_{2}(0)
\end{gathered}
$$

We reshape Eqs.(33), (34) and (35). In this way we attain a set of algebraic equations which enables us to calculate the initial conditions of system (29).

$\Phi_{11}(h) \operatorname{col}_{1}(0)+\left(\Phi_{12}(h)-1\right) \operatorname{col}_{2}(0)+\Phi_{13}(h) \operatorname{col}_{1}(0)+\Phi_{14}(h) \operatorname{col}_{2}(0)=0$ 


$$
\begin{gathered}
\left(\Phi_{31}(h)-1\right) \operatorname{col}_{1}(0)+\Phi_{32}(h) \operatorname{col}_{2}(0)+\Phi_{33}(h) \operatorname{col}_{1}(0)+\Phi_{34}(h) \operatorname{col}_{2}(0)=0 \\
\Phi_{41}(h) \operatorname{col}_{1}(0)+\Phi_{42}(h) \operatorname{col}_{2}(0)+\left(\Phi_{43}(h)-1\right) \operatorname{col}_{1}(0)+\Phi_{44}(h) \operatorname{col}_{2}(0)=0 \\
\operatorname{col}_{2}(h)=\Phi_{21}(h) \operatorname{col}_{1}(0)+\Phi_{22}(h) \operatorname{col}_{2}(0)+\Phi_{23}(h) \operatorname{col}_{1}(0)+\Phi_{24}(h) \operatorname{col}_{2}(0) \\
\left(A_{0}^{T} \otimes I+I \otimes A_{0}^{T}\right) \operatorname{col}_{1}(0)+\left(I \otimes A_{1}^{T}\right) \operatorname{col}_{2}(0)+\left(A_{1}^{T} \otimes I\right) \operatorname{col}_{2}^{T}(0)+ \\
+\left(I \otimes A_{2}^{T}\right) \operatorname{col}_{2}(h)+\left(A_{2}^{T} \otimes I\right) \operatorname{col}_{2}^{T}(h)=-\operatorname{colW}
\end{gathered}
$$

\section{Formulation of the parametric optimization problem}

Let us consider a time-delay system with a P-controller

$$
\left\{\begin{array}{l}
\frac{d x(t)}{d t}=\sum_{j=0}^{m} A_{j} x\left(t-h_{j}\right)+B u(t-h) \\
u(t)=-P x(t) \\
x\left(t_{0}+\theta\right)=\varphi(\theta)
\end{array}\right.
$$

for $t \geqslant t_{0}, \theta \in[-h, 0]$, where $x(t) \in \mathbb{R}^{n}$ is the state of system (42), $u(t) \in \mathbb{R}^{p}$ is the control, $A_{j} \in \mathbb{R}^{n \times n}, B \in \mathbb{R}^{n \times p}, P \in \mathbb{R}^{p \times n}$ is a P-controller gain, $\varphi \in P C\left([-h, 0], \mathbb{R}^{n}\right)$ is the initial function, $0=h_{0}<h_{1}<\ldots<h_{m}=h$ are delays.

System (42) can be written in an equivalent form

$$
\left\{\begin{array}{l}
\frac{d x(t)}{d t}=\sum_{j=0}^{m} A_{j} x\left(t-h_{j}\right)-B P x(t-h) \\
x\left(t_{0}+\theta\right)=\varphi(\theta)
\end{array}\right.
$$

for $t \geqslant t_{0}, \theta \in[-h, 0]$.

In parametric optimization problem will be used the performance index of quality

$$
J=\int_{t_{0}}^{\infty} x^{T}\left(t, t_{0}, \varphi\right) W x\left(t, t_{0}, \varphi\right) d t
$$

where $W \in \mathbb{R}^{n \times n}$ is a symmetric positive definite matrix and $x\left(t, t_{0}, \varphi\right)$ is a solution of Eq. (43) for initial function $\varphi$.

Problem. Determine the matrix $P \in \mathbb{R}^{p \times n}$ whose minimize an integral quadratic performance index of quality (44).

According to Eq. (6) the value of the performance index of quality (44) is equal to the value of the functional (10) for initial function $\varphi$. To calculate the value of the functional (10) we need a Lyapunov matrix $U(\xi)$. To obtain a Lyapunov matrix $U(\xi)$ we have to solve a system of equations (11), (12) and (13). 


\section{Parametric optimization for a scalar system with two delays}

Let us consider a system with two delays and a P-controller

$$
\left\{\begin{array}{l}
\frac{d x(t)}{d t}=a x(t)+b x(t-h)+c x(t-2 h)+u(t-2 h) \\
u(t)=-p x(t) \\
x(\theta)=\varphi(\theta)
\end{array}\right.
$$

$t \geqslant 0, x(t) \in \mathbb{R}$ is the state of system (45), $u(t) \in \mathbb{R}$ is the control, $\varphi(\theta)$ for $\theta \in[-2 h, 0]$ is the initial function, $0 \leqslant h, 2 h$ are time delays, the parameter $p$ is a gain of a P-controller.

One can reshape Eq. (45) to a form

$$
\left\{\begin{array}{l}
\frac{d x(t)}{d t}=a x(t)+b x(t-h)+(c-p) x(t-2 h) \\
x(\theta)=\varphi(\theta)
\end{array}\right.
$$

for $t \geqslant 0$ and $\theta \in[-2 h, 0]$.

In parametric optimization problem we use the performance index of quality

$$
J=\int_{0}^{\infty} w x^{2}(t, \varphi) d t
$$

where $w>0$ and $x(t, \varphi)$ is a solution of Eq. (46) for initial function $\varphi$.

The Lyapunov functional for system (46) has a form, see formula (10)

$$
\begin{gathered}
v(\varphi)=U(0) \varphi^{2}(0)+2 b \varphi(0) \int_{-h}^{0} U(-\theta-h) \varphi(\theta) d \theta+ \\
+2(c-p) \varphi(0) \int_{-h}^{0} U(-\theta-2 h) \varphi(\theta) d \theta+b^{2} \int_{-h}^{0} \int_{-h}^{0} U(\theta-\eta) \varphi(\theta) \varphi(\eta) d \eta d \theta+ \\
+2 b(c-p) \int_{-h-2 h}^{0} \int_{-h+\theta-\eta) \varphi(\theta) \varphi(\eta) d \eta d \theta+(c-p)^{2}}^{0} \int_{-2 h-2 h}^{0} \int_{-2 h}^{0} U(\theta-\eta) \varphi(\theta) \varphi(\eta) d \eta d \theta
\end{gathered}
$$

The value of the performance index of quality (47) is equal to the value of the functional (48) for initial function $\varphi$

$$
J=v(\varphi)
$$


To obtain the value of the performance index of quality one needs a Lyapunov matrix $U(\xi)$ for $\xi \in[0,2 h]$. In Chapter 4 was presented a method of determination of the Lyapunov matrix for a system with two delays.

System of equations (27) takes a form

$$
\left[\begin{array}{c}
\frac{d}{d \xi} U_{1}(\xi) \\
\frac{d}{d \xi} U_{2}(\xi) \\
\frac{d}{d \xi} Z_{1}(\xi) \\
\frac{d}{d \xi} Z_{2}(\xi)
\end{array}\right]=G\left[\begin{array}{l}
U_{1}(\xi) \\
U_{2}(\xi) \\
Z_{1}(\xi) \\
Z_{2}(\xi)
\end{array}\right]
$$

where

$$
G=\left[\begin{array}{cccc}
a & 0 & b & c-p \\
b & a & c-p & 0 \\
-b & -c+p & -a & 0 \\
-c+p & 0 & -b & -a
\end{array}\right]
$$

Initial conditions of system (50) one obtains solving the algebraic equation

$$
Q\left[\begin{array}{c}
U_{1}(0) \\
U_{2}(0) \\
Z_{1}(0) \\
Z_{2}(0)
\end{array}\right]=\left[\begin{array}{c}
0 \\
0 \\
0 \\
-w
\end{array}\right]
$$

where

$$
Q=\left[\begin{array}{cccc}
\Phi_{11}(h) & \Phi_{12}(h)-1 & \Phi_{13}(h) & \Phi_{14}(h) \\
\Phi_{31}(h)-1 & \Phi_{32}(h) & \Phi_{33}(h) & \Phi_{34}(h) \\
\Phi_{41}(h) & \Phi_{42}(h) & \Phi_{43}(h)-1 & \Phi_{44}(h) \\
p_{41} & p_{42} & p_{43} & p_{44}
\end{array}\right]
$$

and

$$
\begin{aligned}
& p_{41}=2 a+2(c-k) \Phi_{21}(h) \\
& p_{42}=2 b+2(c-k) \Phi_{22}(h) \\
& p_{43}=2(c-k) \Phi_{23}(h) \\
& p_{44}=2(c-k) \Phi_{24}(h) .
\end{aligned}
$$


$\Phi(\xi)$ is a fundamental matrix of solutions of Eq. (50) given by (32). Let us consider the initial function $\varphi$ given by a formula

$$
\varphi(\theta)= \begin{cases}x_{0} & \text { for } \theta=0 \\ 0 & \text { for } \theta \in[-2 h, 0)\end{cases}
$$

where $x_{0} \in \mathbb{R}$ is an initial state of system (46). The value of performance index of quality is equal to the value of functional (48) for $\varphi$ given by formula (53) and is given by a term

$$
J=v(\varphi)=U(0) x_{0}^{2}
$$

We search for an optimal gain which minimize the index (54) for given $x_{0}=1$, $w=1, a=-1, b=-0.5$ and $c=1$. Fig. 1 shows the value of the index $J(p)$ for $a=-1$, $b=-0.5, c=1$ and $h=1$. You can see that there exists a critical value of the gain $p_{\text {crit }}$. The system (46) is stable for gains less then critical one and unstable for gains grater then critical.

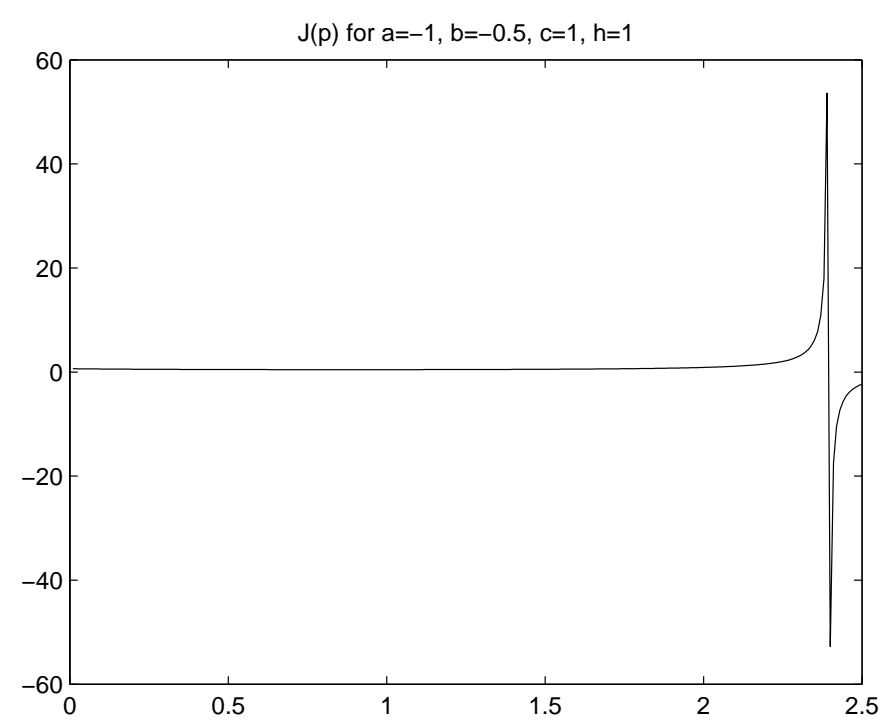

Figure 1: $\mathrm{J}(\mathrm{p})$, p belongs to interval $[0, \mathrm{p} 1]$, where $\mathrm{p} 1>$ pcrit

Fig. 2 shows the value of the index $J(p)$ for $a=-1, b=-0.5, c=1, h=1$ and for $p$ less then critical gain. You can see that the function $J(p)$ is convex and has a minimum.

Optimization results are given in Tab. 1.

From Tab. 1 implies that a critical gain $p_{\text {crit }}$ depends on the value of time delay. Fig. 3 shows the graphs of functions $U_{1}(\xi), U_{2}(\xi), Z_{1}(\xi)$ and $Z_{2}(\xi)$ obtained with the Matlab code, for parameters of system (46) used in optimization process for $p=2$. 


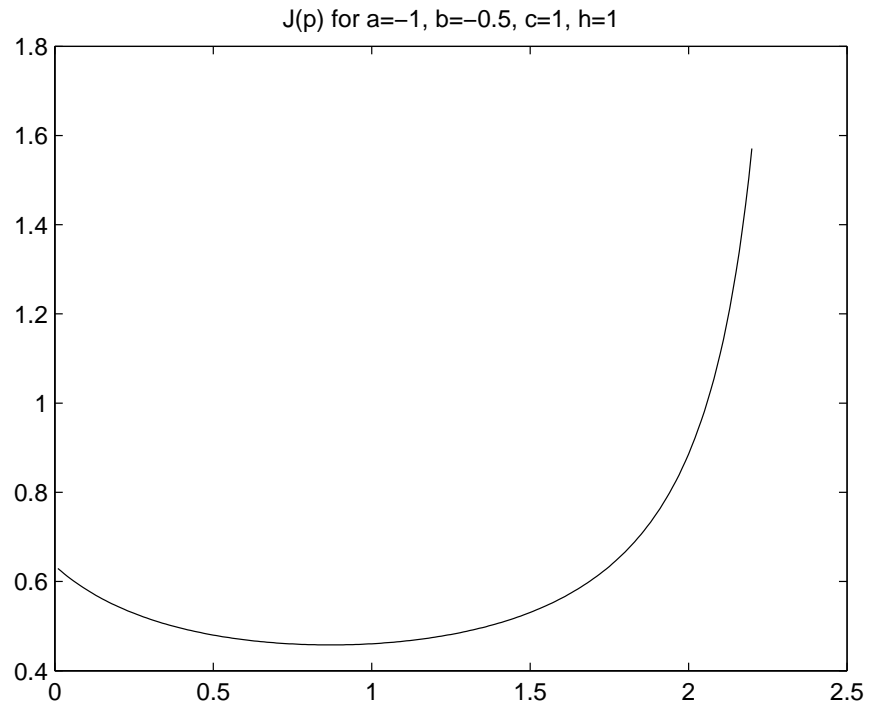

Figure 2: $\mathrm{J}(\mathrm{p})$, p belongs to interval $[0, \mathrm{p} 1]$, where $\mathrm{p} 1<\mathrm{p}_{\text {crit }}$

Table 12: Optimization results

\begin{tabular}{|c|c|c|c|}
\hline Delay h & Optimal gain & Critical gain & Index value \\
\hline \hline 0.5 & 1.15 & 3.13 & 0.4043 \\
\hline 1.0 & 0.87 & 2.39 & 0.4578 \\
\hline 1.5 & 0.90 & 2.17 & 0.4964 \\
\hline 2.0 & 0.96 & 2.07 & 0.5252 \\
\hline 2.5 & 1.02 & 2.03 & 0.5428 \\
\hline
\end{tabular}

\section{Conclusions}

In the paper a Lyapunov matrices approach to the parametric optimization problem of time-delay systems with two delays is presented. The value of integral quadratic performance index of quality is equal to the value of the Lyapunov functional for the initial function of time-delay system. The Lyapunov functional is determined by means of the Lyapunov matrix. The paper can be used in determination of the value of optimal gain which minimize ISE (Integral of Squared Error) for systems with two delays. Using formulas presented in Chapter 6 and a simple Matlab code it is possible to obtain the value of optimal gain for required parameters of system (46). 


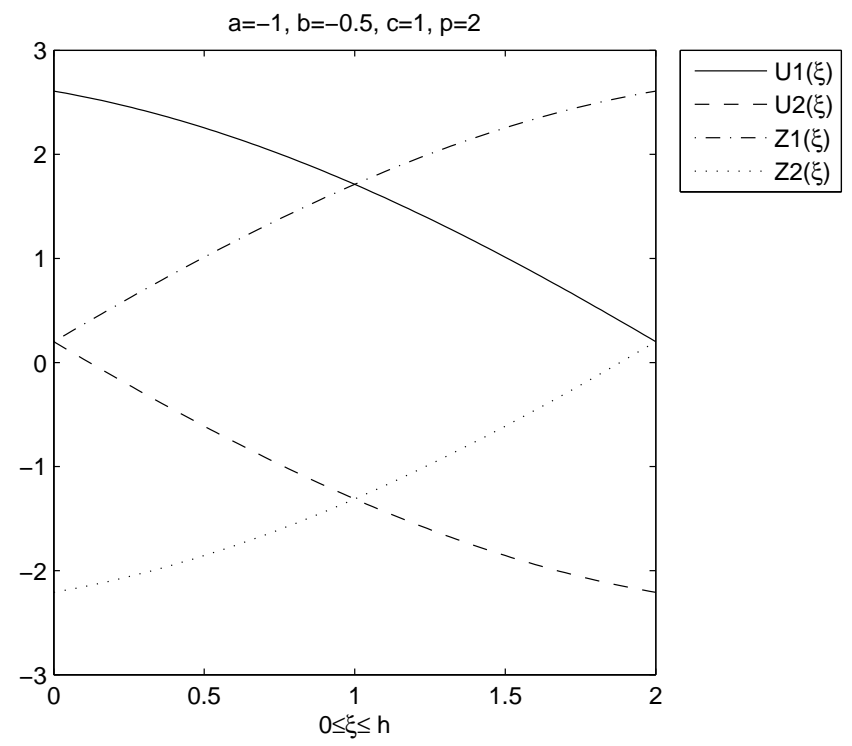

Figure 3: $U_{1}(\xi), U_{2}(\xi), Z_{1}(\xi), Z_{2}(\xi)$ for $\mathrm{p}=2$

\section{References}

[1] R. Bellman, K. Cooke: Differential-difference equations, New York,Academic Press, (1963).

[2] J. DudA: Lyapunov functional for a linear system with two delays. Control and Cybernetics, 39 (2010), 797-809.

[3] J. DUDA: Lyapunov functional for a system with k-non-commensurate neutral time delays. Control and Cybernetics, 39 (2010), 1173-1184.

[4] J. DudA: Lyapunov functional for a system with a time-varying delay. Int. J. of Applied Mathematics and Computer Science, 22 (2012), 327-337.

[5] J. DudA: A Lyapunov functional for a neutral system with a time-varying delay. Bulletin of the Polish Academy of Sciences Technical Sciences, 61 (2013), 911-918.

[6] J. DUDA: Lyapunov matrices approach to the parametric optimization of timedelay systems. Archives of Control Sciences, 25 (2015), 279-288.

[7] J. DUDA: A Lyapunov functional for a neutral system with a distributed time delay. Mathematics and Computers in Simulation, 119 (2016), 171-181.

[8] J. DUDA: Lyapunov matrices approach to the parametric optimization of a neutral system. Archives of Control Sciences, 26 (2016), 81-93. 
[9] H. Górecki, S. Fuksa, P. Grabowski, A. Korytowski: Analysis and Synthesis of Time Delay Systems. John Wiley \& Sons. Chichester, New York, Brisbane, Toronto, Singapore 1989.

[10] V.L. KHARITONOV: Lyapunov functionals and Lyapunov matrices for neutral type time delay systems: a single delay case. Int. J. of Control, 78 (2005), 783-800.

[11] V.L. KhARITONOV: Lyapunov matrices for a class of neutral type time delay systems. Int. J. of Control, 81 (2008), 883-893.

[12] V.L. KHARITONOV: On the uniqueness of Lyapunov matrices for a time-delay system. Systems \& Control Letters, 61 (2012), 397-402.

[13] YU. M. REPIN: Quadratic Lyapunov functionals for systems with delay. Prikl. Mat. Mekh., 29 (1965), 564-566.

[14] S. Rodriguez, V.L. Kharitonov, J. Dion, L. Dugard: Robust stability of neutral systems: a Lyapunov-Krasovskii constructive approach. Int. J. of Robust and Nonlinear Control, 14 (2004), 1345-1358. 